\title{
ON OPTIMAL THIRD ORDER ROTATABLE DESIGNS
}

\author{
NORMAN R. DRAPER \\ Department of Statistics, University of Wisconsin-Madison, Madison, WI 53706, USA
}

\section{Berthold Heiligers}

Fakultät für Mathematik, Universität Magdeburg, D-39016 Magdeburg, Germany

\section{FRIEDRICH PUKELSheIM}

Institut für Mathematik, Universität Augsburg, D-86135 Augsburg, Germany

(Received November 7, 1994; revised May 15, 1995)

\begin{abstract}
We obtain results for choosing optimal third order rotatable designs for the fitting of a third order polynomial response surface model, for $m \geq 3$ factors. By representing the surface in terms of Kronecker algebra, it can be established that the two parameter family of boundary nucleus designs forms a complete class, under the Loewner matrix ordering. In this paper we first narrow the class further to a smaller complete class, under the componentwise eigenvalue ordering. We then calculate specific optimal designs under Kiefer's $\phi_{p}$-criteria (which include the often used E-, A-, and D-criteria). The E-optimal design attains a particularly simple, explicit form.
\end{abstract}

Key words and phrases: Complete classes of designs, design efficiency, E-, A-, D-optimal designs, response surface designs, third order models. 


\section{Introduction}

The idea of making a design rotatable is a useful one in practice. It enables the experimental information to be obtained equally in all directions, at the same distance from the origin, in the space of real design variables $x_{1}, x_{2}, \ldots, x_{m}$. Third order rotatability assumes that it is desired to fit a cubic polynomial in $x_{1}, x_{2}, \ldots, x_{m}$ to the available experimental data.

There are several ways of representing the third order model. Draper and Pukelsheim (1994) choose a Kronecker representation because rotatability of any order is then easily handled using the methods of multilinear algebra. Although the Kronecker representation is redundant, in that it repeats such terms as mixed products, the redundancy is of the same quality as in a dispersion matrix: We preferably think of the latter as a matrix, albeit symmetric, rather than replacing it through the set of functionally independent terms on and above the diagonal.

While we find the dispersion matrix analogy persuasive, and the handling of the Kronecker representation model encouraging, we admittedly deviate from traditional paths. Traditionally, higher order response surfaces are expressed in the Schläflian notation, or using the Box-Hunter minimal set of monomials. Draper, Gaffke and Pukelsheim (1991), in their Section 9, compare these three approaches to rotatability, to the effect that (1) the three corresponding variance surfaces all coincide, (2) rotatability is the same whether defined in one way or the other, and (3) reparametrization arguments furnish a way to re-express these approaches as linear transformations of one another.

Hence we find it fair to say that the three approaches serve the practical statistical purpose in an identical manner. However, the associated technicalities are not identical, due to the fact that the moment matrices $M_{K}, M_{S}, M_{B H}$ of the Kronecker, Schläflian, or Box-Hunter representations differ. It is precisely because of these technical differences that the eigenvalue decomposition of the Kronecker representation moment matrix $M_{K}$ takes an almost explicit form, as in (3.27) of Draper and Pukelsheim (1994). The same could also be achieved under the Schläflian calculus, but we find the details less transparent. A similar eigenvalue decomposition for the Box-Hunter moment matrix $B_{B H}$ is not available, see Section 2 of Galil and Kiefer (1979).

For these reasons, and since we believe that the choice of the statistical model is at the discretion of the statistician rather than being forced upon us by nature, we employ the Kronecker representation model. The minor technical difficulty that the moment matrix $M_{K}$ is rank deficient is of no relevance, in an age of generalized inverses. For 
example, Kiefer's $\phi_{p}$-optimality criteria are simply taken to be the mean of order $p$ of the positive eigenvalues of $M_{K}$. For a detailed discussion of this issue see Section 8.18 in Pukelsheim (1993).

Draper and Pukelsheim (1994) discuss the pattern of the moment matrices associated with third order designs under the Kronecker representation. A conclusion emerging from that work is that a complete class of designs that provides third order rotatability consists of the family of boundary nucleus designs, reviewed in Section 2. These designs only depend on a weight parameter $\alpha$ and a radius parameter $r$.

In Section 3, we narrow the set of boundary nucleus designs by excluding certain values of $\alpha$ and $r$, for a given dimension $m$. This applies to all optimality criteria that depend on the design only through the eigenvalues of its moment matrix.

In Section 4, we specify our optimality criteria as those in the family of matrix means, $\phi_{p}$, with $-\infty \leq p \leq 1$. This family of criteria was introduced by Kiefer (1974), and is discussed in detail in Pukelsheim (1993). Here we calculate the parameter values $\alpha(p)$ and $r(p)$ that are optimal under the criterion $\phi_{p}$, as a function of $p \in[-\infty, 1]$. This includes numeric results for the classic E-, A-, and D-criteria, for $p=-\infty,-1,0$, respectively.

For E-optimality, the numerical evidence suggests - and we verify in Section 5that the optimal weight $\alpha(-\infty)$ has a simple closed form expression, and that the optimal radius is $r(-\infty)=1 / 2$. Section 6 concludes with some numerical efficiency comparisons to link our results with previous work.

The practical conclusions from the present work are that the optimal radius $r(p)$ varies little with $p$ but stays close to $1 / 2$ while, however, the weight $\alpha(p)$ depends considerably on $p$.

\section{Boundary nucleus designs}

Boundary nucleus designs depend on a weight parameter $\alpha \in[0,1]$ and a radius parameter $r \in[0,1]$. They allocate weight $\alpha$ to the boundary sphere of radius $\sqrt{m}$ while placing the remaining weight, $1-\alpha$, on a concentric inner sphere nucleus of radius $r \sqrt{m}$. The radius of the boundary sphere is chosen as $\sqrt{m}$ so that this sphere contains all points of the form $( \pm 1, \pm 1, \ldots, \pm 1)$. Hence the present class contains the two level full or fractional factorial designs that are basic to the subject, see Box and Draper (1987). Replacement of the uniform distribution on the sphere by point sets that have 
identical moments up to and including order six would provide a design which can be practically implemented.

The moment matrix of boundary nucleus designs is given by equation (3.27) in Draper and Pukelsheim (1994). From this we can obtain the eigenvalues as functions of the weight $\alpha$ and the radius $r$, for dimension $m \geq 3$, as follows:

$$
\begin{aligned}
& \theta_{1}(\alpha, r)=\frac{2 m}{m+2}\left[\alpha+(1-\alpha) r^{4}\right] \\
& \theta_{2}(\alpha, r)=\frac{1}{2}\left\{m\left[\alpha+(1-\alpha) r^{4}\right]+1\right\}+\frac{1}{2} \sqrt{u(\alpha, r)}, \\
& \theta_{3}(\alpha, r)=\frac{1}{2}\left\{m\left[\alpha+(1-\alpha) r^{4}\right]+1\right\}-\frac{1}{2} \sqrt{u(\alpha, r)}, \\
& \theta_{4}(\alpha, r)=\frac{6 m^{2}}{(m+2)(m+4)}\left[\alpha+(1-\alpha) r^{6}\right], \\
& \theta_{5}(\alpha, r)=\frac{1}{2}\left\{\frac{3 m^{2}}{m+2}\left[\alpha+(1-\alpha) r^{6}\right]+\left[\alpha+(1-\alpha) r^{2}\right]\right\}+\frac{1}{2} \sqrt{v(\alpha, r)}, \\
& \theta_{6}(\alpha, r)=\frac{1}{2}\left\{\frac{3 m^{2}}{m+2}\left[\alpha+(1-\alpha) r^{6}\right]+\left[\alpha+(1-\alpha) r^{2}\right]\right\}-\frac{1}{2} \sqrt{v(\alpha, r)},
\end{aligned}
$$

where the functions $u$ in $\theta_{2}$ and $\theta_{3}$, and $v$ in $\theta_{5}$ and $\theta_{6}$ are given by

$$
\begin{aligned}
& u(\alpha, r)=\left\{m\left[\alpha+(1-\alpha) r^{4}\right]-1\right\}^{2}+4 m\left[\alpha+(1-\alpha) r^{2}\right]^{2}, \\
& v(\alpha, r)=\left\{\frac{3 m^{2}}{m+2}\left[\alpha+(1-\alpha) r^{6}\right]-\left[\alpha+(1-\alpha) r^{2}\right]\right\}^{2}+\frac{12 m^{2}}{m+2}\left[\alpha+(1-\alpha) r^{4}\right]^{2} .
\end{aligned}
$$

The multiplicities of the eigenvalues are, respectively, $n_{1}=\frac{1}{2} m(m+1)-1, n_{2}=n_{3}=1$, $n_{4}=\frac{1}{6} m(m+1)(m+2)-m$, and $n_{5}=n_{6}=m$.

Theorem 4.1 and Corollary 4.2 of Draper and Pukelsheim (1994) show that any design, rotatable or not, can be improved upon by an appropriate boundary nucleus design, with respect to a wide class of optimality criteria. Furthermore, Theorem 4.1 implies that the boundary nucleus designs form a complete class under the Loewner matrix ordering, while Lemma 2 of Heiligers and Schneider (1992) then entails that this is actually a minimal complete class. Their result holds within the class of all designs, whereas the related Theorem X.7.5 of Karlin and Studden (1966) applies only within the class of rotatable designs. Kiefer (1961) appears to be among the first dealing with optimality of rotatable designs in the context of the approximate theory, in his Section 3.2.

The class of all nucleus boundary designs can be further narrowed when, in addition, we consider the componentwise ordering of the eigenvalues $\theta_{1}, \theta_{2}, \ldots, \theta_{6}$. 


\section{A complete class result under eigenvalue monotonicity}

If the optimality criterion depends only on the eigenvalues and is isotonic in each of them, as is true for the $\phi_{p}$ family, we would like to exploit the monotonicity properties of the eigenvalues over the $(\alpha, r)$ region, the unit square $[0,1] \times[0,1]$.

The boundaries of this region determine designs that are degenerate for a third order model. The West boundary consists of designs on a single sphere of radius $r \sqrt{m}$, with $r$ growing from zero to one. All of the North and East boundary corresponds to the constant design on the $\sqrt{m}$ sphere.

The South boundary consists of central composite designs, with a growing fraction $\alpha$ of design points out on the $\sqrt{m}$ sphere and a decreasing fraction $1-\alpha$ of center points. These are the second order designs for which eigenvalue monotonicity is discussed in Section 15.19 of Pukelsheim (1993); the eigenvalues $\lambda_{1}(\alpha)$ and $\lambda_{4}(\alpha)$ from that section here take the form $\theta_{2}(\alpha, 0)$ and $\theta_{3}(\alpha, 0)$.

For third order models, interest is in proper two sphere designs, that is, in the interior $(0,1) \times(0,1)$ of the unit square, where the eigenvalues $\theta_{1}, \theta_{2}$, and $\theta_{4}, \theta_{5}$ are seen to be strictly increasing in both $\alpha$ and $r$.

The surface $\theta_{3}$ is a descending ridge, running from its highest point at $\left(\frac{2}{m+4}, 0\right)$ on the South boundary - see the behavior of $\lambda_{4}(\alpha)$ in Exhibit 15.1 of Pukelsheim (1993) down to zero on the North, East, and West boundaries. In the unit square, the position of the ridge is defined by the curve $(f(r), r)$ with $0 \leq r \leq 1$, where

$$
f(r)=\frac{m r^{2}\left(1+r^{2}\right)+2}{m\left(1+r^{2}\right)^{2}+4} .
$$

As $r$ increases from zero to one, $f$ increases from $2 /(m+4)$ to $1 / 2$.

The surface $\theta_{6}$ is a unimodal mound tied down to zero on all boundaries of the square. As we move across $\alpha$ values from West to East, with $r$ fixed, it rises steeply out of the origin to a maximum at $g(r)$, where

$$
g(r)=\frac{r}{1+r} \frac{3 m^{2} r^{2}\left(1-r+r^{2}\right)+m+2}{3 m^{2}\left(1-2 r+3 r^{2}-2 r^{3}+r^{4}\right)+m+2} .
$$

As $r$ increases from zero to one, $g$ increases from zero to $1 / 2$.

For $r \in[0,1]$ we have $g(r) \leq f(r)$. Thus, traversing from West to East, the $g$ ridge is met prior to the $f$ ridge. Therefore it is the ridge $g$ that splits the unit square into a West region and an East region such that, if a pair $(\alpha, r)$ lies in the West region, 
then the corresponding design is improved upon - in terms of increasing eigenvaluesby moving from $\alpha$ to $g(r)$. That is, the radius $r$ is maintained, and the weight $\alpha$ is increased to the ridge value $g(r)$. Hence the East region determines a complete class of designs, under all optimality criteria which depend on the eigenvalues of the moment matrix in an isotonic manner. Figure 1 shows the two regions, for selected dimensions $m$.

Figure 1 about here

The ridge curves $g$ show little practical variation as $m$ increases from 3 onwards. As $m$ tends to infinity, the limiting curve is

$$
h(r)=\frac{r^{3}}{1+r} \frac{1-r+r^{2}}{1-2 r+3 r^{2}-2 r^{3}+r^{4}} .
$$

In Figure 1 it is included as the curved line that lies inside the shaded West region, identical in the four subplots.

\section{4. $\phi_{p}$-Optimal designs}

Specific design calculations can be made for any optimality criterion, as a function of the two parameters $\alpha$ and $r$. For the Kiefer $\phi_{p}$ family, the criterion is the mean of order $p$ of the $\left(\begin{array}{c}m+3 \\ 3\end{array}\right)$ positive eigenvalues of the moment matrix,

$$
\phi_{p}(\alpha, r)=\left(\frac{6}{(m+1)(m+2)(m+3)} \sum_{j=1}^{6} n_{j} \theta_{j}^{p}(\alpha, r)\right)^{1 / p}
$$

Numerical calculations for $-10 \leq p \leq 0.5$ now give optimal values $\alpha(p)$ and $r(p)$. The ensuing curves for dimensions $m=3,4,5$, and 10 appear in Figure 1 . The solutions for E-, A-, and D-optimality, corresponding to $p=-10,-1,0$, have been noted by bold dots. The tick marks on the line $r=1 / 2$ indicate the optimal weights $\alpha(p)$. They are carried over from one subplot to the next, and thus illustrate the movement of these values as a function of the dimension $m=3,4,5,10$. 


\section{E-Optimality}

Although E-optimality is closely approximated by $p=-10$, strictly speaking it requires $p=-\infty$. Indeed, Figure 1 suggests the definite answer. For instance, for $m=10$ the point with coordinates $\alpha(-10)=0.12592$ and $r(-10)=0.50003$ is visually identical to the point with $g(1 / 2)=0.12586$ and $1 / 2$ on the bounding ridge $g$. The residual numerical difference seems to account for the remaining range $p \in[-\infty,-10]$.

Therefore we conjecture the E-optimal parameters to be

$$
\alpha(-\infty)=g\left(\frac{1}{2}\right)=\frac{1}{3} \frac{9 m^{2}+16 m+32}{27 m^{2}+16 m+32}
$$

and $r(-\infty)=1 / 2$. Once the conjecture is seen, it is easy to provide a stringent proof for it. It plainly follows from the facts that (i) the mound $\theta_{6}$ has its unique peak at $(\alpha(-\infty), 1 / 2)$, and that (ii) at this point the other eigenvalues $\theta_{1}, \ldots, \theta_{5}$ are larger than $\theta_{6}$. The E-optimal value is $\theta_{6}(\alpha(-\infty), 1 / 2)=3 m /\left(27 m^{2}+16 m+32\right)$.

\section{Efficiencies of alternative designs}

The efficiency of an alternative design $\xi$ is defined to be $\phi_{p}(\xi) / v(p)$, where $v(p)$ is the $\phi_{p}$-optimal value. That is, $v(p)$ is the $\phi_{p}$-criterion value of the design belonging to the optimal parameter values $\alpha(p)$ and $r(p)$. Thus efficiency-usually quoted in percent - is a number indicating to what extent the alternative design exhausts the maximum possible information, as measured by the given criterion function.

It is noticeable that the $r(p)$ values show little variation over the whole range of $p$, being slightly larger than $1 / 2$. At the same time $\alpha(p)$ takes a wide range of values, roughly between 0.1 and one. Hence the radius of the smaller sphere remains approximately constant at $\sqrt{m} / 2$, but the weight $\alpha(p)$ increases with $p$. It would appear that optimizing $\alpha$ alone while keeping $r=1 / 2$ fixed leads to designs of high efficiency.

This is confirmed by our numerical calculations for dimensions $m=3,4,5,10$. Under the D-criterion $(p=0)$, the efficiencies of the D-optimal $r=1 / 2$ designs all are above 99.65 percent. For the A-criterion $(p=-1)$, the efficiencies of the A-optimal $r=1 / 2$ designs decrease monotonically, being 99.3., 98.7, 98.3, 97.6 percent. Close to the E-criterion $(p=-10)$, practically full efficiency 100 percent is reached.

Furthermore our results compare favorably with those of Gaffke and Heiligers (1995a,b) and Galil and Kiefer (1979). Those authors employ the usual, Box-Hunter 
representation of the response surface. Also their outer sphere radius is one, rather than $\sqrt{m}$ as used by us. We recall that our choice would enable the experimenter to include, in the design, the full factorial design $2^{m}$ or fractions thereof.

For our efficiency comparison relative to the Kronecker representation optimal value $v(p)$, we therefore include the designs that are $\phi_{p}$-optimal in the Box-Hunter model, again for dimensions $m=3,4,5,10$. Of course, full efficiency of 100 percent obtains under the D-criterion, because of its invariance properties. For other criteria $(p \neq 0), \phi_{p}$-optimality under the Box-Hunter representation does not necessitate rotatability. Hence we have to distinguish whether we refer optimality to the full set of all designs, or to the proper subset of rotatable designs.

Under the A-criterion, the Box-Hunter A-optimal designs in the full set of all designs have efficiencies of 96, 95, 94, 90 percent; the Box-Hunter A-optimal designs in the subset of rotatable designs are 97, 96, 95, 91 percent efficient, respectively. See also Gaffke and Heiligers (1995a). Close to the E-criterion $(p=-10)$, the first corresponding set of efficiencies is $92,93,9496$ percent, for the second set all efficiencies lie between 95 and 96 percent. See also Gaffke and Heiligers (1995b).

\section{Acknowledgments}

We thank Claudia Heyne and Michael Vorleuter, students at the University of Augsburg, whose "Numerik Praktikum" computations have been quoted in this paper. N.R.D. and F.P. would like to acknowledge partial support from the Alexander-vonHumboldt-Stiftung through a Max-Planck-Award for cooperative research. N.R.D. is grateful for partial support from the Scientific and Environmental Affairs Division of the North Atlantic Treaty Organization, and from the National Security Agency through Grant MDA904-95-H-1020. 


\section{REFERENCES}

Box, G.E.P. and Draper, N.R. (1987). Empirical Model-Building and Response Surfaces, Wiley, New York.

Draper, N.R. and Pukelsheim, F. (1994). On third order rotatability, Metrika, 41, $137-161$.

Draper, N.R., Gaffke N. and Pukelsheim, F. (1991). First and second order rotatability of experimental designs, moment matrices, and information surfaces, Metrika, 38, 129-161.

Gaffke, N. and Heiligers, B. (1995a). Optimal and robust invariant designs for cubic multiple regression, Metrika, 42, 29-48.

Gaffke, N. and Heiligers, B. (1995b). Computing optimal approximate invariant designs for cubic regression on multidimensional balls and cubes, Journal of Statistical Planning and Inference, forthcoming.

Galil, Z. and Kiefer, J.C. (1979). Extrapolation designs and $\Phi_{p}$-optimum designs for cubic regression on the $q$-ball, Journal of Statistical Planning and Inference, $\mathbf{3}$, $27-38$.

Heiligers, B. and Schneider, K. (1992). Invariant admissible and optimal designs in cubic regression on the $\nu$-ball, Journal of Statistical Planning and Inference, 31, 113-125.

Karlin, S. and Studden, W.J. (1966). Tchebycheff Systems: With Applications in Analysis and Statistics, Wiley Interscience, New York.

Kiefer, J.C. (1961). Optimum experimental designs V, with applications to systematic and rotatable designs, in Proceedings of the Fourth Berkeley Symposium on Mathematical Statistics and Probability, (Ed. J. Neyman), University of California, Berkeley CA, 1, 381-405.

Kiefer, J.C. (1974). General equivalence theory for optimum designs (approximate theory), Annals of Statistics, 2, 849-879.

Pukelsheim, F. (1993). Optimal Design of Experiments, Wiley, New York. 


$$
g(r)
$$

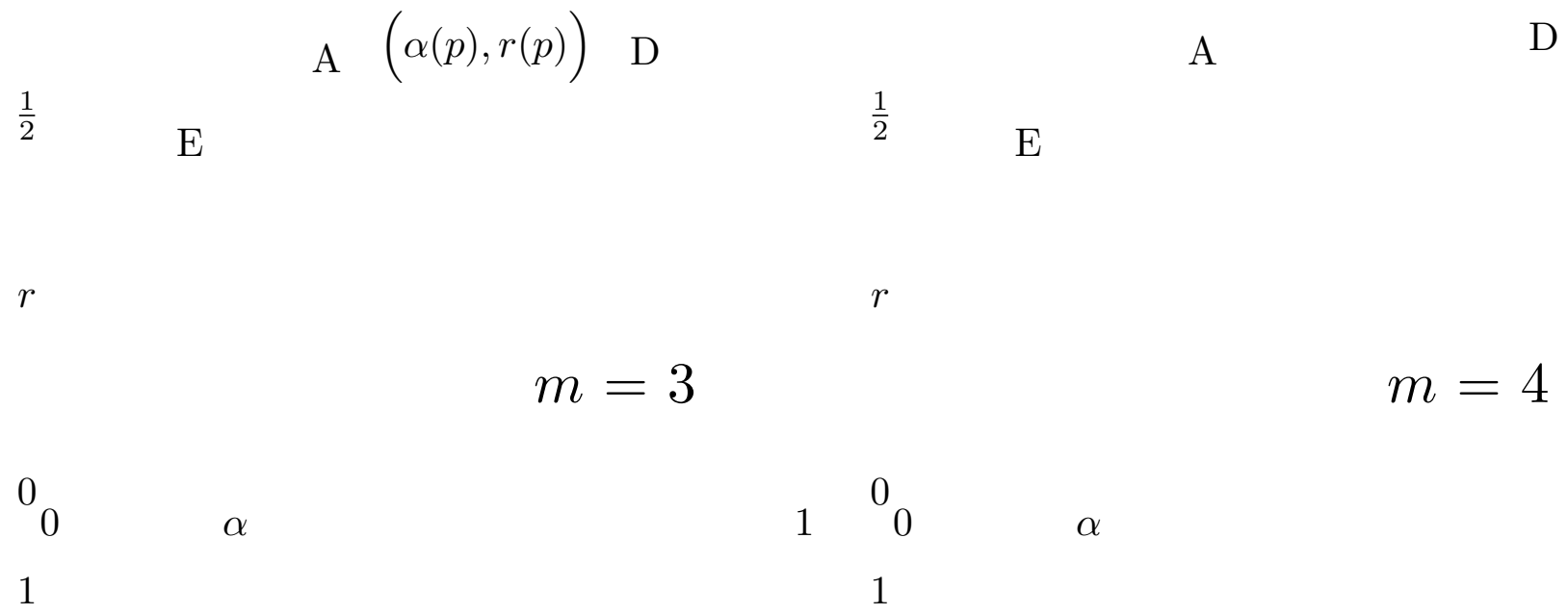

A

$\mathrm{D}$

Figure 1. $\phi_{p}$-Optimal Weight and Radius, for Dimensions $m=3,4,5,10$. In the grey Western region, a horizontal shift to the bounding curve $g(r)$ improves the eigenvalues. The curve in the white Eastern region represents the $\phi_{p}$-optimal weights $\alpha(p)$ and radii $r(p)$, for $-10 \leq p \leq 0.5$, with dots marking E-, A-, and D-optimality. 